\title{
QUALIDADE E MATURAÇÃO DE TANGERINAS E SEUS HÍBRIDOS EM SÃO PAULO ${ }^{1}$
}

\author{
SIMONE RODRIGUES DA SILVA², JOÃO CARLOS DE OLIVEIRA³, \\ EDUARDO SANCHES STUCHI ${ }^{4}$, EDUARDO TOLLER REIFF ${ }^{5}$
}

RESUMO - A ampliação da oferta de tangerinas tanto no mercado interno como no externo é limitada pela grande quantidade de sementes nos frutos das principais cultivares, danos na casca resultante de doenças e pela pequena produção nas épocas mais quentes do ano. O presente trabalho teve como objetivo caracterizar a qualidade dos frutos e a época de maturação de 46 tangerinas e híbridos em condições de campo na Estação Experimental de Citricultura de Bebedouro (EECB), Bebedouro-SP. As variedades de tangerinas e híbridos (tangores e tangelos) foram introduzidas de bancos de germoplasma da Itália, Portugal, Espanha e França, e a pesquisa desenvolveu-se durante os anos agrícolas de 2001/2002 e 2002/2003. Foram avaliadas características externas de qualidade, como altura e diâmetro dos frutos, massa dos frutos, facilidade em descascar com a mão e características internas como: número de sementes, teor de sólidos solúveis totais, acidez total titulável, rendimento em suco, "ratio" e rendimento em polpa. Duas cultivares apresentaram frutos de boa qualidade e época de maturação precoce, podendo ser disponibilizadas no mercado, num período de escassez deste tipo de citros, e serem alternativas à tangerina- ‘Cravo'. Quatro cultivares que apresentaram boa qualidade e maturação mediana poderão ser alternativas à 'Poncã' .

Termos para indexação: Cítrus, variedades, sólidos solúveis, mercado.

\section{QUALITY AND MATURATION OF TANGERINES AND THEIR HYBRIDS IN SÃO PAULO}

\begin{abstract}
The extension of tangerine offering both for internal and external markets is limited by the large amount of seeds present in the main cultivars, the occurrence of peel injures caused by diseases and the small fruit production during the hottest periods of the year. This work was aimed to characterize the fruit quality and maturation period of 46 tangerines and hybrids grown in the field at the Estação Experimental de Citricultura de Bebedouro (EECB), located in Bebedouro county, in São Paulo State, Brazil. Tangerines and their hybrids (tangors and tangelos) were introduced from germplasm banks of Italy, Portugal, Spain and France, and the study was conducted during the years of 2001/2002 and 2002/2003. External fruit quality was assessed by measurements of fruit height and diameter, fruit mass and peeling facility, as well as the internal quality characteristics, such as seed number, total soluble solids content, total titrable acidity, juice content, ratio and pulp content. Two cultivars produced good quality and early ripening fruits that may be offered in the local market in periods of low fruit offer, as alternatives to the 'Cravo' tangerine. Four cultivars that presented good quality and a moderate maturation precocity may be introduced as alternatives for the 'Ponkan' cultivar.
\end{abstract}

Index terms: Citrus, varieties, total soluble solids content, market.

\footnotetext{
'(Trabalho 282-08). Recebido em: 13-11-2008. Aceito para publicação em: 23-06-2009.

${ }^{2}$ Departamento de Produção Vegetal - ESALQ/USP, Caixa Postal 9, CEP 13418-970, Piracicaba-SP, srsilva@esalq.usp.br

${ }^{3}$ Prof. Dr. Depto. Produção Vegetal - Fitotecnia - FCAV/UNESP, Jaboticabal-SP, jocaoliv@fcav.unesp.br

${ }^{4}$ Pesquisador Embrapa Mandioca e Fruticultura Tropical, Bebedouro-SP, stuchi@estacaoexperimental.com.br

${ }^{5}$ Estação Experimental de Citricultura de Bebedouro, Bebedouro-SP, eduardoreiff@estacaoexperimental.com.br
} 


\section{INTRODUÇÃO}

O Brasil destina grande parte da produção de citros ao processamento de suco e, embora seja considerado o maior produtor de citros do mundo, com 15,9 milhões de toneladas, pouco menos de 1,3 milhão de toneladas correspondem a tangerinas e híbridos. A China, quarto maior produtor de citros, é líder na produção de tangerinas, sendo responsável por 52\% da produção mundial (FNP, 2009).

Dentre as principais variedades de tangerinas e híbridos de interesse econômico no Brasil, estão as tangerinas-'Cravo' e 'Poncã', a mexerica'Rio' e o tangor-'Murcott'. A tangerina-“Poncã” (C. reticulata Blanco) e o tangor "Murcott" [C. sinensis (L.) Osbeck x C. reticulata Blanco] correspondem a 80\% do cultivo deste grupo de frutas cítricas no Estado de São Paulo (Amaro \& Caser, 2003).

Os frutos da 'Poncã' apresentam de 5-8 sementes, peso médio de $138 \mathrm{~g}$, 43\% de rendimento em suco, $10,8^{\circ}$ Brix, 0,85\% de acidez e ratio médio de 12,7 (Pio et al., 2005). Essas características conferem boa qualidade aos frutos e permitem sua aceitação no mercado de frutas frescas. Entretanto, essa cultivar apresenta alternância de produção, produzindo excessivamente num ano e pouco no outro.

O tangor-'Murcott' apresenta frutos com grande quantidade de sementes, em média 20, peso médio de 140 g, $48 \%$ de rendimento em suco, $12,6^{\circ}$ Brix, $0,92 \%$ de acidez e ratio médio de 13,7 , sendo destinado tanto para o mercado de frutas frescas, interno e externo, como para a indústria (Pio et al., 2005).

Apesar de a produção brasileira de tangerina ser a quarta maior do mundo, há grande limitação para a exportação de suas frutas, por não atenderem a padrões de qualidade exigidos pelos principais mercados internacionais, como baixo número de sementes e ausência de danos na casca (Boteon, 2003).

A introdução de novas cultivares com características que atendam às expectativas do consumidor, como tamanho, forma, cor e sabor adequados, pequeno número de sementes, resistência a doenças e oferta de frutos nas épocas mais quentes do ano é fundamental para o crescimento do setor produtivo.

O presente trabalho objetivou a caracterização da qualidade dos frutos e época de maturação de 46 tangerinas e híbridos.

\section{MATERIAL E MÉTODOS}

O trabalho foi conduzido na Estação Experimental de Citricultura de Bebedouro (EECB), situada no município de Bebedouro-SP, Brasil (latitude 2053'16” S, longitude 48 $28^{\prime} 11^{\prime \prime}$ W, clima Cwa: subtropical-inverno moderado e seco, verão quente e chuvoso). Variedades de tangerinas (Citrus reticulata Blanco) e híbridos (tangores: Citrus reticulata Blanco x Citrus sinensis (L.) Osbeck e tangelos: Citrus reticulata x Citrus paradisi Macf), introduzidos de bancos de germoplasma da Itália, Portugal, Espanha e França, com a colaboração da Embrapa, Cenargen e Fundecitrus, foram avaliadas, e entre eles estão as tangerinas e híbridos de tangerinas, listadas a seguir: Zanzibar SRA 442; Macaque SRA 426; Carvalhais Vidigueira,; Antillaise SRA 497; Fewtrell SRA 418; Natal Tightskin SRA 481; Changsha SRA 413; Fortune SRA 31; Wallent SRA 438; Carvalhais (N); Lebon SRA 425; Ponkan Yoshida SRA 585; Late Emperor SRA 423; East Índia SRA 414; Redskin SRA 428; Beuty of Glen Retreat SRA 261; Malvásio SRA 115; Rodeking SRA 431; Page SRA 159; C-54-4-4 SRA 337; À Peau Lisse SRA 267; Encore SRA 190; Temple Sue Linda SRA 467; Fuzhu SRA 599; as clementinas Caffin SRA 385; Reina SRA 534; Commune SRA 92; Ragheb SRA 386; 2kr Monreal; Oroval Y. 45; Tomatera SRA 535; De Nules VCR; Bruno SRA 531; Oroval SRA 335; Commune SRA 88; Nules SRA 389; Commune SRA 85; os tangelos: Nova IVIA - 86 - 2 (N); Allspice SRA 327; Nocatee SRA 452; Thornton Vero SRA 460; o tangor Clemelin IVIA 355, e as satsumas Saigon SRA 227, Kowano SRA 167, Miyagawa SRA 444, e Salzara SRA 341 (N - representa material de origem nucelar).

As mudas enxertadas em limoeiro- 'Cravo' foram plantadas em um solo classificado como Argissolo Vermelho-Amarelo típico, textura média/ argilosa A moderado (PVA), cujos atributos químicos podem ser vistos no Quadro 1, em fevereiro de 2000, no espaçamento de 7,0 x 3,0 metros e conduzidas de acordo com os tratos culturais recomendados por Donadio et al. (1998). O pomar foi dividido em parcelas de três plantas para cada genótipo. No primeiro ano, foram aplicados $250 \mathrm{~g} /$ metro linear de calcário dolomítico (PRNT 100\%) e 450 g de superfosfato simples no sulco de plantio, além de 2 ton/ha do mesmo calcário em área total e adubações com nitrocálcio, na dose de $60 \mathrm{~g} /$ planta, e da fórmula 10-10-10, na dose de 200g/planta. No segundo ano, foram realizadas duas adubações: $200 \mathrm{~g} /$ planta de $12-$ 06-12 (junho) e 200g/planta de 20-00-10 (dezembro); 
no terceiro ano: 200 g/planta de 20-00-10 (março) e 500 g/planta de 20-10-10 (outubro), e no quarto ano: 500g/planta da fórmula 20-10-10 (fevereiro).

A qualidade dos frutos foi avaliada no laboratório da EECB, nos anos de 2002 e 2003, e de cada parcela, de três plantas, foram coletados cinco frutos. Dados de temperatura e precipitação são apresentados na Tabela 1.

As características externas avaliadas foram: a) altura e diâmetro dos frutos: medidos com uma régua tipo calha graduada em centímetros; b) massa dos frutos: determinada através de uma balança digital, com seus valores expressos em gramas; c) facilidade em descascar com a mão: avaliação feita manualmente, caracterizando o descascamento dos frutos como fácil (nota 3), regular (nota 2) e difícil (nota 1). Esta classificação foi elaborada comparativamente à facilidade encontrada para se descascarem manualmente as principais variedades comerciais, isto é; 'Poncã’ e ‘Cravo’ (3), Tangelos 'Orlando’ e ‘Nova' (2) e 'Murcott' (1).

Internamente, foram avaliados: a) número de sementes: contado após a extração do suco de cinco frutos, obtendo-se o número médio de sementes por fruto; b) teor de sólidos solúveis totais (SST): determinado por refratometria a $20^{\circ} \mathrm{C}$ em refratômetro de leitura direta, e expressos em ${ }^{\circ} \mathrm{Brix}$; c) determinação da acidez total titulável (ATT): foram diluídos $20 \mathrm{~mL}$ de suco em $100 \mathrm{~mL}$ de água destilada. Depois, utilizando-se de uma bureta digital, foi realizada a titulação da solução com $\mathrm{NaOH}$ a 0,3125N, expressa em g de ácido cítrico/100 mL de suco; d) rendimento em suco: determinado através dos valores da relação entre o peso do suco/peso dos frutos, expresso em \%; e) "ratio": determinado pela relação aritmética entre os sólidos solúveis (SS) e a acidez titulável (AT), e f) rendimento em polpa: determinado através dos valores da relação entre o peso da polpa/peso dos frutos expresso em \%.

Cada genótipo foi avaliado até atingir o “ratio" igual ou superior a 12 (Steger, 1990). Em 2003, além das características de qualidade comuns a 2002, foram avaliados o rendimento em polpa e a facilidade de descascamento, utilizando-se de nova amostra de cinco frutos.

\section{RESULTADOS E DISCUSSÃO}

O tipo de solo sob o qual o trabalho foi conduzido, ofereceu condições físicas adequadas para o desenvolvimento radicular profundo das plantas, assim como grande capacidade de armazenamento de água, o que permitiu uma boa qualidade de frutos, principalmente se comparado a solos arenosos
(Castle, 1995).

\section{Características externas de qualidade Altura e diâmetro dos frutos}

A relação entre altura e diâmetro do fruto é utilizada para definir o formato do mesmo. No ano de 2002, apenas as variedades Miyagawa SRA 444, Page SRA 159 e Thornton Vero SRA 460 apresentaram relação entre altura e diâmetro maior que 1. O mesmo aconteceu para as variedades 'Caffin SRA 385', 'Carvalhais Vidigueira’ e ‘Carvalhais’ no ano de 2003 (Tabela 2). A maioria das variedades avaliadas apresentou relação menor que 1 , indicando que possuem as mesmas proporções do formato do fruto das variedades de tangerinas comerciais, ou seja, forma oblata.

\section{Massa dos frutos}

Segundo Pio et al. (2005), a tangerina'Cravo' apresentou massa média de 135g, a Mexerica-do-rio 133g, a Poncã 138g e o tangor'Murcott' 140g. Em 2002, as variedades Miyagawa SRA 444, Nules SRA 389; C-54-4-4 - SRA 337 e o Thornton Vero SRA 460 produziram frutos com massas superiores às encontradas para as variedades comerciais. Oliveira et al. (2005) observaram para a cultivar 'Clementina de Nules', na região do Rio Grande do Sul, frutos com peso médio de 157,1 g; enquanto Borges et al. (2008) relataram uma massa média em torno de 140 g. Possivelmente, uma pequena produção de frutos da 'Clementina de Nules’, neste trabalho, tenha resultado no maior peso dos mesmos. Já as variedades 'Bruno SRA 531'; 'Commune SRA 85' e 'Commune SRA 88'; 'East Índia SRA 414’; 'Redskin SRA 428'; 'Fuzhu SRA 599'; 'Temple Sue Linda SRA 467'; 'Zanzibar SRA 442' e o Clemelin 'IVIA 355' apresentaram valores de massa abaixo do relatado por Pio et al. (2005) para as variedades comerciais, porém seus diâmetros são aceitáveis segundo a Classificação das Tangerinas (Ceagesp, 2000), que considera como mínimos valores de 5,0 e 5,2 cm para o menor e o maior diâmetro, respectivamente. As demais variedades avaliadas apresentaram massa média similar às das principais variedades comerciais (Tabela 2).

Em 2003, Satsumas 'Saigon SRA 227'; 'Miyagawa SRA 444' e 'Kowano SRA 167', as Tangerinas 'Page SRA 159'; 'Poncã Yoshida SRA 585’; 'Beuty Of Glen Retreat SRA 261'; 'C-54-4-4 SRA 337', 'Lebom SRA 425'; 'Late Emperor SRA 423’, ‘À Peau Lisse SRA 267’ e o Tangelo ‘Allspice SRA 327' também produziram frutos com massas superiores aos relatados por Pio et al. (2005) para as variedades comerciais. As variedades Clementina Bruno SRA 531; Caffin SRA 385; Commune SRA 
88; Ragheb SRA 386 e 2 Kr Monreal; Tangerina Changsha SRA 413; Carvalhais Vidigueira e Carvalhais apresentaram valores de massa abaixo do relatado por Pio et al. (2005) para as variedades comerciais, porém seus diâmetros são aceitáveis, segundo a Classificação das Tangerinas (Ceagesp, 2000). As demais variedades avaliadas apresentaram massa média similar às das principais variedades comerciais (Tabela 2).

Na região de Valência, na Espanha, a massa média para a variedade 'Nova' foi de 115,1 g (Bono, 1994). Borges \& Pio (2003) encontraram massa média superior à descrita na Espanha para a variedade Nova, o que também ocorreu neste trabalho.

\section{Facilidade de descascamento}

A maioria das variedades foram classificadas com nota 3 , ou seja, são fáceis de descascar, e essa facilidade que ocorreu em todas as satsumas e clementinas avaliadas, estão de acordo com os dados apresentados por Oliveira et al. (2005). Já as variedades Carvalhais; Fewtrell SRA 418; Page SRA 159 e Wallent SRA 438 foram classificadas com nota 2, apresentando-se como regulares no descascamento, quando comparadas com padrões comerciais dos tangelos Nova e Orlando. Apenas a Tangerina 'C-54-4-4 SRA 337' e o Tangelo 'Allspice SRA 327' foram classificados com nota 1 , ou difíceis de descascar, se comparados com o padrão dado pelo tangor-'Murcott' e tangores de modo geral. Embora a maioria dos tangelos sejam classificados com nota 2, o 'Allspice SRA 327' foi uma exceção neste trabalho, apresentando-se como de difícil descascamento (Tabela 2). Cabe salientar que, mesmo sendo considerado difícil de descascar, o tangor-'Murcott' tem boa aceitação por consumidores e pela indústria de sucos, sendo assim, as variedades semelhantes não teriam problema de aceitação.

\section{Características internas de qualidade Número de sementes}

No geral, as variedades apresentaram elevado número de sementes (Tabela 2), provavelmente devido à proximidade de variedades polinizadoras. No tangelo-Nova IVIA-86-2 foram encontradas em média 19,8 sementes/fruto. Ferraro et al. (2006) observaram que frutos de tangelo-Nova, resultantes da polinização natural cruzada, apresentaram em média 24,5 sementes/fruto.

O menor número de sementes foi observado nas variedades de Satsuma 'Miyagawa SRA 444'; 'Saigon SRA 227', 'Kowano SRA 167’ e 'Salzara
SRA 341'; Tangelo 'Nocatee SRA 452' e 'Allspice SRA 327'; Clementina 'Tomatera SRA 535' e 'Caffin SRA 385; 'Ragheb SRA 386'; e Tangerina 'Fewtrell SRA 418' e 'Fortune SRA 31'. Nas satsumas, o pólen não é fértil, o que contribui para a produção de frutos sem sementes (Hodgson, 1967). Domingues et al. (1999), estudando diversos acessos de tangerinas e híbridos do Banco Ativo de Germoplasma de Citros do Centro de Citricultura, observaram para a Satsuma Unshiu Wase, uma média de 1,3 semente por fruto.

\section{Sólidos Solúveis Totais (SST)}

No ano de 2002, apenas as variedades 'Miyagawa SRA 444', 'Tomatera SRA 535', 'Oroval SRA 335' e 'Commune SRA 85’ apresentaram baixos valores de SST, entre $6,30-8,00^{\circ}$ Brix, abaixo do mínimo recomendado para as variedades comerciais, que é de 9,0 Brix (Ceagesp, 2000). Em 2003, as variedades Bruno SRA 531; Caffin SRA 385; Ragheb SRA 386; 2kr Monreal; Oroval; Saigon 227; Miyagawa SRA 444, Kowano SRA 167; Salzara SRA 341; Changsha SRA 413; Y.45; Natal Tightskin SRA 481; Fuzhu SRA 599 e Late Emperor SRA 423 também apresentaram frutos com valores de SST abaixo do recomendado pelo Ceagesp (2000). Os valores de SST observados para as duas introduções de Clementina de Nules, nos dois anos de avaliação, foram menores que o relatado por Oliveira et al. (2005) para esta variedade, nas condições do Rio Grande do Sul, o que pode ser explicado pela maior temperatura na região avaliada. No entanto, esses valores foram superiores ao mínimo proposto pelo Ceagesp para as variedades comerciais.

Mourão Filho et al. (2007), avaliando dois híbridos de tangerinas quanto à qualidade dos frutos, verificaram que, quando enxertados em limoeiro Cravo, os frutos dessas variedades apresentaram os menores valores de SST, ainda acima do mínimo recomendado pelo Ceagesp. O mesmo foi observado por Espinoza-Núñez et al. (2008), na avaliação da qualidade de frutos da tangerina-Fairchild sobre limoeiro-Cravo.

\section{Acidez Total Titulável (ATT)}

Pio et al. (2005) relataram valores médios de ATT de 0,80\% para 'Cravo', 0,99\% para Mexericado-’Rio’, 0,85\% para 'Poncã' e 0,92\% para 'Murcott'. Entre as variedades avaliadas, observou-se variação na ATT de 0,41 a $1,14 \%$ (2002) e de 0,40 a $1,04 \%$ (2003), com a maioria das variedades apresentando valores abaixo dos relatados por Pio et al. (2005). Todos os genótipos de satsumas avaliados mostraram valores de ATT variando de 0,51 a 0,58\% em 2002 e de 0,54 a 0,58\% em 2003 (Tabela 2). Esses dados 
concordam em parte com Cyrillo (2000) que, avaliando 64 seleções de satsumas, considerou como aceitáveis valores de ATT entre 0,55 a 0,81\% para todas as variedades que estudou.

\section{Ratio}

A queda de frutos em algumas variedades impediu a continuidade das avaliações, pois tiveram de ser colhidas com "ratio" inferior ao adotado como referência. Tanto em 2002 como 2003, a maioria das variedades avaliadas apresentou valores de "ratio" superiores a 12, o que indica que teriam boa aceitação pelos consumidores, pois enquadram-se dentro da faixa de "ratio", de 12 a 15, em que, segundo Steger (1990), os frutos cítricos receberam as maiores notas quanto ao sabor em avaliações sensoriais.

\section{Rendimento em suco}

Em 2002, essa característica variou de 22,44 a $52,10 \%$, enquanto, em 2003, a variação foi de 12,35 a 54,25\%. Nas Satsumas a variação foi de 26,40 a $35,26 \%$ (2002) e de 29,15 a 38,20\% (2003), enquanto nas Clementinas esta variação foi de 27,61 a 41,79\% (2002) e de 12,35 a 46,53\% (2003) (Tabela 2). A maioria das variedades avaliadas apresentou valores de rendimento abaixo dos relatados por Pio et al. (2005) para as variedades comerciais (40 a 48\%) e pela Classificação de Tangerinas (Ceagesp 2000), na qual o rendimento em suco pode ser de 35\% para Poncã e Mexerica-do-rio, 40\% para Cravo e 42\% para Murcott.

Considerando apenas as variedades avaliadas nos dois anos de trabalho com valores de rendimento entre 35 e 48\% (Pio et al., 2005; Ceagesp, 2000), destacam-se com valores adequados para a característica as Clementinas 'Nules VCR', 'Bruno SRA 531' e 'Commune SRA 85' e as tangerinas 'Encore SRA 190' e 'Temple Sue Linda SRA 467'. Oliveira et al. (2005) encontraram para a Clementina de Nules, cultivada na região do Rio Grande do Sul, rendimento em suco de 52,8\%, muito acima dos observados neste trabalho para as duas introduções avaliadas, o que poderia ser explicado pela interferência do clima nesta característica de qualidade.

\section{Rendimento em polpa}

Na maioria das variedades, o rendimento em polpa variou de 62,6\% a 79,03\%, valores que são próximos à Poncã Yoshida (67,7\%). Apenas a Satsuma 'Saigon SRA 227' apresentou baixo rendimento em polpa, 32,8\% (Tabela 2).

\section{Época de maturação dos frutos}

Na Tabela 3, encontram-se as variedades avaliadas, de acordo com a sequência em que atingiram o ponto de colheita ("ratio" 12) em 2002 e 2003.

Tanto a Clementina-de-Nules VCR como a Satsuma-Miyagawa SRA 444 apresentaram maturação precoce, entre os meses de fevereiromarço. Além da precocidade, as variedades destacaram-se pelo rendimento em polpa, que foi superior à Poncã-Yoshida (67,7\%) e pela facilidade no descascamento. Essas duas características são importantes na aceitação de frutos pelo mercado consumidor. Oliveira et al. (2005) relataram a facilidade do descascamento em frutos da Clementina-de-Nules. Miyagawa SRA 444 destacouse também pelo pequeno número de sementes (Tabela 2). Essas características permitiriam a colheita e a comercialização de seus frutos antes da variedade 'Cravo', cuja maturação varia de precoce à meia-estação (Pio et al., 2005). Nos dois anos de avaliação, não ocorreram diferenças climáticas na fase de desenvolvimento dos frutos, de outubro a março, para as variedades precoces, portanto as diferenças observadas nos períodos de maturação não podem ser atribuídas às condições climáticas.

Donadio et al. (1998) relataram que a época de produção da 'Clementina', no Estado de São Paulo, varia de meados de fevereiro a maio, e que estas épocas estariam associadas tanto a cultivares mais precoces quanto às mais tardios dentro do grupo, enquanto as Satsumas produziriam entre os meses de janeiro a março.

Foram observadas diferenças entre as épocas de maturação de um ano para o outro para algumas variedades. Um exemplo extremo foi a Clementina'Commune SRA 88' e a Satsuma-'Saigon SRA 227' mais tardias em 2002 (maturação em maio) que em 2003 (maturação em março). Já a tangerina'Rodeking SRA 431' apresentou-se mais precoce em 2002 (maturação em maio) que em 2003 (maturação em setembro). Por outro lado, certas variedades apresentaram semelhanças entre as épocas de maturação, nos dois anos de avaliação, como as tangerinas-'Beuty Of Glen Retreat SRA 261'; 'Temple Sue Linda SRA 467', 'Fuzhu SRA 599', 'À Peau Lisse SRA 267' e a Satsuma 'Miyagawa SRA 444'.

Entre as variedades que apresentaram a mesma época de maturação da 'Poncã' em 2002 e 2003, algumas podem ser alternativas à mesma, já que os valores médios de todas as características avaliadas são similares aos relatados por Pio et al. (2005) e aos preditos pela Classificação das 
Tangerinas (Ceagesp, 2000). Destacam-se as variedades: Commune SRA 85 para rendimento em suco, rendimento em polpa superior ao observado para Poncã- Yoshida $(67,7 \%)$ e facilidade no descascamento; Page SRA 159 por apresentar valores adequados de SST, rendimento em suco e polpa; Encore SRA 190 e Temple Sue Linda SRA 467 com valores de rendimento em suco adequado, facilidade de descascamento e rendimento em polpa superior à Poncã-Yoshida (67,7\%).

Donadio et al. (1998) relataram um período de produção entre os meses de janeiro e março para as satsumas em São Paulo. No presente trabalho, as satsumas tiveram o período de produção mais amplo, estendendo-se até maio em 2002 e até abril em 2003, o que nem sempre seria interessante por coincidir com a 'Poncã'.

A tangerina-'Malvasio SRA 115' mostrouse tardia nos dois anos de avaliação. Em 2002, não apresentou frutos para avaliações posteriores a 18-09-02, data da última avaliação, quando seu “ratio” foi inferior a 10,0. Já em 2003, a mandarina'Malvasio SRA 115' atingiu o índice de maturação proposto em 24-09-03.
Genótipos cujas épocas de maturação não foram definidas, tiveram sua colheita antecipada, pela queda de frutos, ou por não apresentarem frutos nas amostragens seguintes.

A maioria das variedades avaliadas atingiu a maturação entre os meses de abril e junho (2002) e entre março e junho (2003). Considerando os genótipos que apresentaram a mesma época de maturação nos dois anos de avaliação, destacamse como precoces as variedades De Nules VCR e Miyagawa SRA 444 por estarem maduras entre os meses de fevereiro e março. Nos meses de verão, há escassez deste tipo de citros no mercado, logo poderiam ser alternativas à 'Cravo'. Entre as variedades de meia-estação, destacam-se pelas características de qualidade as variedades Commune SRA 85, Page SRA 159, Encore SRA 190 e Temple Sue Linda SRA 467 que poderiam ser alternativas à 'Poncã', com maturação entre abril-junho. Nenhuma variedade apresentou maturação tardia, ou seja, posterior ao tangor-'Murcott’.

TABELA 1 - Dados de temperatura máxima e mínima $\left({ }^{\circ} \mathrm{C}\right)$ e precipitação (mm) nos anos de 2001 a 2003. Bebedouro-SP, Brasil.

\begin{tabular}{|c|c|c|c|c|c|c|c|c|c|c|c|}
\hline \multirow{4}{*}{ MÊS } & \multicolumn{11}{|c|}{ ANOS } \\
\hline & \multicolumn{3}{|c|}{2001} & \multicolumn{4}{|c|}{2002} & \multirow{3}{*}{ MÊS } & \multicolumn{3}{|c|}{2003} \\
\hline & T. min. & T. máx. & $\mathrm{P}$ & MÊS & T. min. & T. máx. & $\mathrm{P}$ & & T. min. & T. máx. & $\mathrm{P}$ \\
\hline & $\left({ }^{\circ} \mathrm{C}\right)$ & $\left({ }^{\circ} \mathrm{C}\right)$ & $(\mathrm{mm})$ & & $\left({ }^{\circ} \mathrm{C}\right)$ & $\left({ }^{\circ} \mathrm{C}\right)$ & $(\mathrm{mm})$ & & $\left({ }^{\circ} \mathrm{C}\right)$ & $\left({ }^{\circ} \mathrm{C}\right)$ & $(\mathrm{mm})$ \\
\hline JAN & 19,35 & 33,31 & 106,80 & JAN & 19,70 & 31,85 & 292,10 & JAN & 20,9 & 31,0 & 379,50 \\
\hline FEV & 20,10 & 33,43 & 164,10 & FEV & 19,69 & 31,43 & 308,70 & FEV & 20,3 & 33,6 & 212,90 \\
\hline MAR & 19,19 & 33,62 & 89,40 & MAR & 19,62 & 34,16 & 171,20 & MAR & 19,3 & 31,9 & 194,90 \\
\hline ABR & 18,94 & 33,27 & 16,40 & ABR & 18,02 & 34,28 & 0,70 & ABR & 17,4 & 31,0 & 148,20 \\
\hline MAI & 14,83 & 28,15 & 82,10 & MAI & 15,85 & 30,30 & 18,70 & MAI & 13,0 & 28,0 & 56,13 \\
\hline JUN & 14,70 & 27,79 & 0,00 & JUN & 17,10 & 32,00 & 0,00 & JUN & 12,9 & 30,1 & 4,30 \\
\hline JUL & 14,95 & 30,08 & 5,60 & JUL & 12,98 & 28,96 & 15,80 & JUL & 11,5 & 29,3 & 13,60 \\
\hline AGO & 16,18 & 30,98 & 49,80 & AGO & 16,48 & 32,20 & 58,90 & AGO & 11,7 & 29,4 & 10,30 \\
\hline SET & 17,25 & 32,08 & 49,60 & SET & 15,77 & 29,63 & 76,10 & SET & 15,0 & 33,2 & 31,70 \\
\hline OUT & 18,12 & 31,78 & 163,80 & OUT & 19,65 & 36,29 & 126,00 & OUT & 18,6 & 30,6 & 71,20 \\
\hline NOV & 19,58 & 32,43 & 178,40 & NOV & 19,38 & 32,31 & 221,60 & NOV & 18,9 & 30,2 & 101,30 \\
\hline DEZ & 19,46 & 31,44 & 246,70 & DEZ & 20,37 & 33,10 & 131,20 & DEZ & 20,2 & 31,2 & 160,20 \\
\hline Média & 17,7 & 31,5 & 1152,7 & Média & 17,9 & 32,2 & 1421,0 & Média & 16,7 & 30,8 & 1384,2 \\
\hline
\end{tabular}




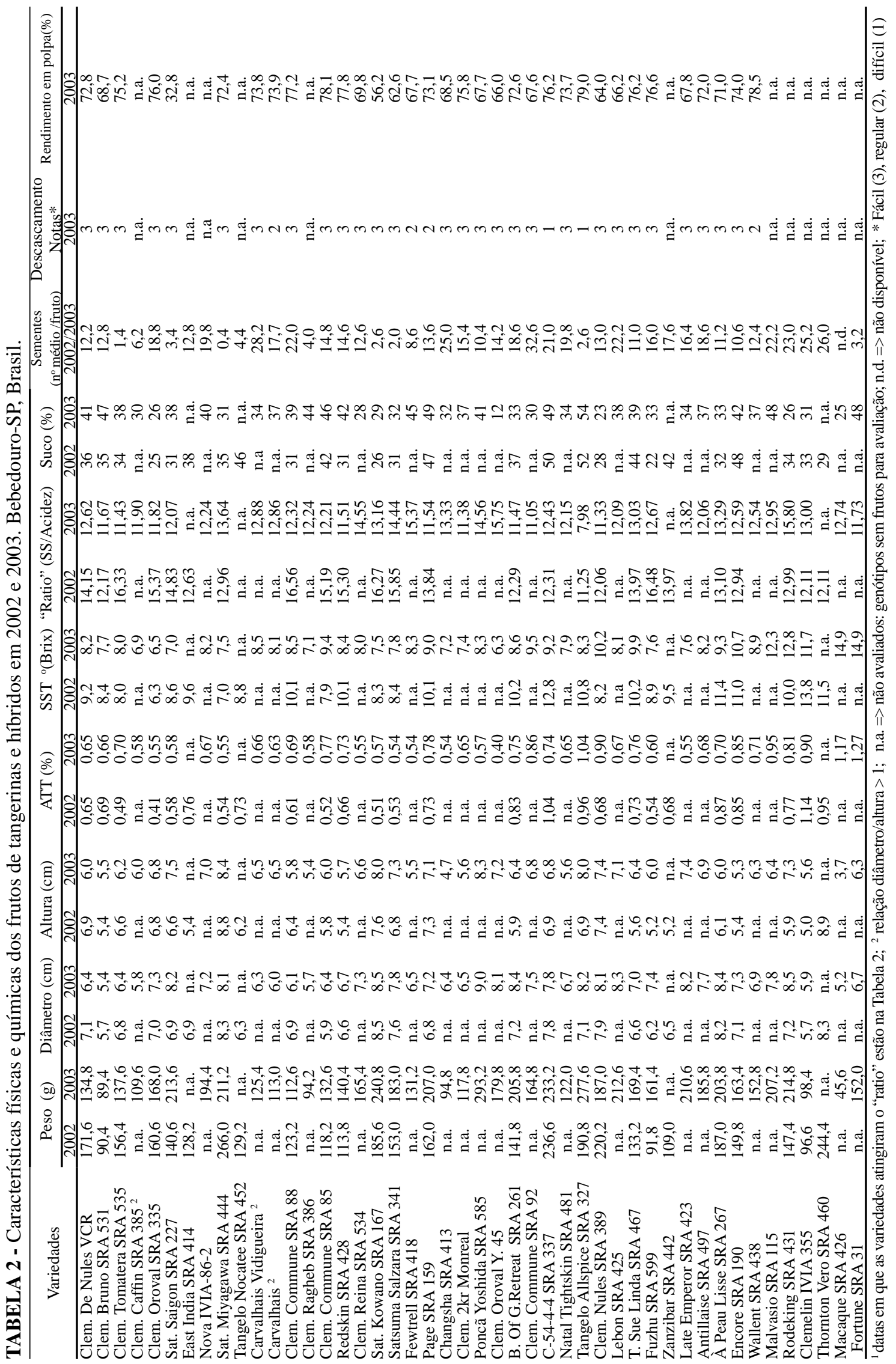



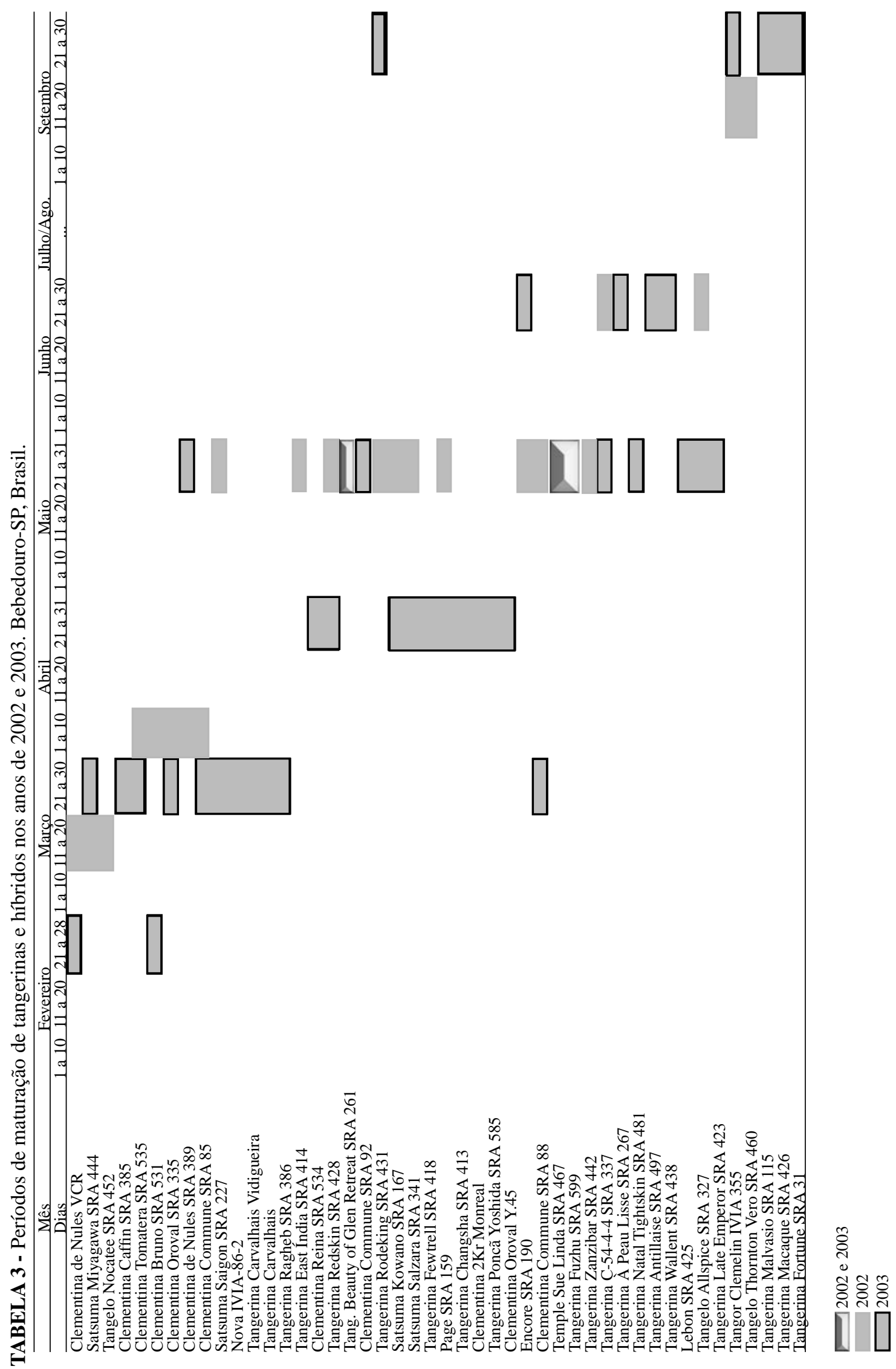

Rev. Bras. Frutic., Jaboticabal - SP, v. 31, n. 4, p. 977-986, Dezembro 2009 
QUADRO 1- Análise química do solo, realizada em junho de 2000, na profundidade de 0-20 cm. BebedouroSP, Brasil.

\begin{tabular}{|c|c|c|c|c|c|c|c|c|c|c|c|}
\hline $\mathrm{pH}$ & M.O. & $\mathrm{P}$ & K & $\mathrm{Ca}$ & Mg & $(\mathrm{H}+\mathrm{Al})$ & S & $\mathrm{Al}$ & SB & $\mathrm{T}$ & $\mathrm{V}$ \\
\hline & & Resina & & & & & & & & & \\
\hline $\mathrm{CaCl} 2$ & $\mathrm{~g} \mathrm{dm}^{-3}$ & $\mathrm{mg} \mathrm{dm}{ }^{-3}$ & \multicolumn{4}{|c|}{$\mathrm{mmol}_{\mathrm{c}} \mathrm{dm}^{-3}$} & $\mathrm{mg} \mathrm{dm} \mathrm{m}^{-3}$ & \multicolumn{3}{|c|}{$\mathrm{mmol}_{\mathrm{c}} \mathrm{dm}^{-3}$} & $\%$ \\
\hline 4,6 & 18 & 3 & 0,3 & 12 & 5 & 33 & -- & -- & 17,3 & 50,3 & 34 \\
\hline
\end{tabular}

\section{CONCLUSÕES}

Duas cultivares apresentaram frutos de boa qualidade e época de maturação precoce, podendo ser disponibilizadas no mercado num período de escassez deste tipo de citros e serem alternativas à tangerina-'Cravo’. Quatro cultivares com boas características de qualidade e maturação mediana poderão ser alternativas à 'Poncã'.

\section{REFERÊNCIAS}

AMARO, A.A.; CASER, D.V. Diversidade do mercado de tangerinas. Informações Econômicas, São Paulo, v.33, n.12, p.51-67, 2003.

AGRIANUAL 2008: anuário estatístico da agricultura brasileira. São Paulo: FNP, 2009. 497 p.

BONO, R. Características de nuevas variedades de agrios. Phytoma, Valência, n.58. p.19-25, 1994.

BORGES, R. S.; OLIVEIRA, R. P.; PIO, R. M.; FARIA, A. P. Catálogo de cultivares de citros de mesa. Pelotas: Embrapa Clima Temperado, 2008. 40 p. (Documentos, 223).

BORGES, R.S.; PIO, R.M. Comparative study of the mandarin hybrid fruit characteristics: Nova, Murcott and Ortanique in Capão Bonito SP, Brazil. Revista Brasileira de Fruticultura, Jaboticabal, v.25, n.3, p.448-452, 2003.

BOTEON, M. Janeiro de pouca laranja. Citricultura Atual, Cordeirópolis, n.32, p.4-5, 2003.
CASTLE, W.S. Rootstock as a fruit quality factor in citrus and deciduous tree crops. New Zealand Journal of Crop and Horticultural Science, Wellington, v.23, n.3, p.383-394, 1995.

CEAGESP. Programa brasileiro para a melhoria dos padrões comerciais e embalagens de hortigranjeiros: classificação das tangerinas. São Paulo, 2000. 8p.

CYRILLO, F.L.L. Avaliação fenotípica de seleções de tangerina “Satsuma” (Citrus unshiu Marcovith) nas condições climáticas da região de Jaboticabal. 2000. 107f. Dissertação (Mestrado em Produção Vegetal) - Faculdade de Ciências Agrárias e Veterinárias, Universidade Estadual Paulista, Jaboticabal, 2000.

DOMINGUES, E.T.; SOUZA, V.C.; SAKURAGUI, C.M.; POMPEU JUNIOR, J.; PIO, R.M.; SOBRINHO, J.T.; SOUZA, J.P. Caracterização morfológica e tangerinas do banco ativo de germoplasma de citros do Centro de Citricultura Sylvio Moreira/IAC. Scientia Agrícola, Piracicaba, v.56, n.1, p. 197-206, 1999.

DONADIO, L.C.; STUCHI, E.S.; CYRILLO, F.L. Tangerinas ou mandarinas. Jaboticabal: FUNEP, 1998. 40 p. (Boletim Citrícola, 5).

ESPINOZA-NÚÑEZ, E.; MOURÃO-FILHO, F.A.A.; STUCHI, E.S.; ORTEGA, E.M.M. Desenvolvimento e produtividade da tangerina "Fairchild" sobre quatro porta-enxertos. Ciência Rural, Santa Maria, v.38, n.6, p.1553-1557, 2008. 
FERRARO, A.E.; PIO, R.M.; AZEVEDO, F.A. Inlfuência da polinização com variedades de laranjadoce sobre o número de sementes de Tangelo Nova. Revista Brasileira de Fruticultura, Jaboticabal, v.28, n.2, p.244-246, 2006.

HODGSON, R.W. Horticultural varieties of citrus. In: REUTHER, W.; WEBBER, H.J.; BATCHELOR, L.D. (Eds.). The citrus industry. Berkeley: University of California, 1967. p. 431-459.

MOURÃO-FILHO, F.A.A.; ESPINOZA-NÚÑEZ, E.; STUCHI, E.S.; ORTEGA, E.M.M. Plant growth, yield, and fruit quality of 'Fallglo' and 'Sunburst' mandarins on four rootstocks. Scientia Horticulturae, Amsterdam, v.114, p.45-49, 2007.
OLIVEIRA, R.P.; CANTILLANO R. F. F.; MALGARIM M. B.; TREPTOW R. O.; GONÇALVES A. S. Características dos citros apirênicos produzidos no Rio Grande do Sul. Pelotas: Embrapa Clima Temperado, 2005. 41 p. (Documentos, 141).

PIO, R. M.; FIGUEIREDO, J.O.; STUCHI, E.S.; CARDOSO, S.A.B. Variedades copas. In: MATTOS JUNIOR, D. et al. (Ed.). Citros. Campinas: Instituto Agronômico/Fundag, 2005. p. 39-60.

STEGER, E. Trinta anos de desenvolvimento em processamento de citros: histórico, estado de arte e visão geral. Laranja, Cordeirópolis, v.11, n.2, p.463-502, 1990. 